\title{
Increased Synthesis of Spermidine as a Result of Upregulation of Arginase I Promotes Axonal Regeneration in Culture and In Vivo
}

\author{
Kangwen Deng, Huifang He, Jin Qiu, Barbara Lorber, J. Barney Bryson, and Marie T. Filbin \\ Biology Department, Hunter College, New York, New York 10065
}

\begin{abstract}
Adult spinal axons do not spontaneously regenerate after injury. However, if the peripheral branch of dorsal root ganglion neurons is lesioned before lesioning the central branch of the same neurons in the dorsal column, these central axons will regenerate and, if cultured, are not inhibited from extending neurites by myelin-associated inhibitors of regeneration such as myelin-associated glycoprotein (MAG). This effect can be mimicked by elevating cAMP and is transcription dependent. The ability of cAMP to overcome inhibition by MAG in culture involves the upregulation of the enzyme arginase I (Arg I) and subsequent increase in synthesis of polyamines such as putrescine. Now we show that a peripheral lesion also induces an increase in Arg I expression and synthesis of polyamines. We also show that the conditioning lesion effect in overcoming inhibition by MAG is initially dependent on ongoing polyamine synthesis but, with time after lesion, becomes independent of ongoing synthesis. However, if synthesis of polyamines is blocked in vivo the early phase of good growth after a conditioning lesion is completely blocked and the later phase of growth, when ongoing polyamine synthesis is not required during culture, is attenuated. We also show that putrescine must be converted to spermidine both in culture and in vivo to overcome inhibition by MAG and that spermidine can promote optic nerve regeneration in vivo. These results suggest that spermidine could be a useful tool in promoting CNS axon regeneration after injury.
\end{abstract}

\section{Introduction}

One major obstacle to spontaneous axonal regeneration after injury in the adult, mammalian CNS is the presence of myelinassociated inhibitors such as myelin-associated glycoprotein (MAG), Nogo A, and oligodendrocyte myelin glycoprotein (OMgp) (Filbin, 2003; Yiu and He, 2006). Both MAG and OMgp as well as one of the inhibitory domains on Nogo A, Nogo66, exert their inhibitory effects by interacting with the NgR:Lingo: p75 receptor complex, resulting in activation of the small GTPase Rho, which is necessary to bring about inhibition. Recently, a second receptor with which MAG, OMgp, and Nogo66 also all interact has been identified, called paired Ig-like receptor B (PirB), a protein first identified on monocytes (Atwal et al., 2008). Other inhibitors of axonal regeneration, such as chondroitin sulfate proteoglycans, are upregulated by reactive astro-

Received March 10, 2009; revised June 16, 2009; accepted June 18, 2009.

This work was supported by National Institutes of Health (NIH) Grant NS37060, the New York State Spinal Cord Injury Research Board, core facility grants from the Research Centers for Minorities Institute/NIH, and Specialized Neuroscience Research Programs/NIH Grant NS41073. We thank Melissa Hilaire for her outstanding technical support, Dr. Lloyd Williams for his help with the image analysis, and Dr. Marco Domeniconi for assistance with this manuscript.

Correspondence should be addressed to Marie T. Filbin, Biology Department, Hunter College, 695 Park Avenue, New York, NY 10065. E-mail: filbin@genectr.hunter.cuny.edu.

K. Deng's present address: Discovery Neuroscience, Wyeth Research, CN8000, Princeton, NJ 08543.

J. Qiu's present address: 11 Horsleydown Mansions, Lafone Street, London SE1 2NA, UK.

B. Lorber's present address: Centre for Brain Repair, University of Cambridge, E. D. Adrian Building, Robinson Way, Cambridge CB2 OPY, UK.

J. B. Bryson's present address: Wolfson Center for Age-Related Diseases, King's College, London SE1 9RT, UK. DOI:10.1523/JNEUROSCI.1175-09.2009

Copyright $\odot 2009$ Society for Neuroscience $\quad$ 0270-6474/09/299545-08\$15.00/0 cytes after injury (Silver and Miller, 2004), and recently a number of repulsive guidance cues that act during development of the nervous system have also been reported to be present at the lesion site in adult animals and/or upregulated after injury (Yiu and $\mathrm{He}, 2006)$. To date, the majority of these inhibitory molecules that could potentially contribute to the lack of axonal regeneration in the adult CNS converge in their signaling on the activation of Rho.

Contrary to the dogma, one situation in which spontaneous axonal regeneration does occur in the adult CNS is of the central branch, the dorsal column axons, of dorsal root ganglion (DRG) neurons, but this only occurs if the peripheral branch of the same neurons has been lesioned 1 week before (Neumann and Woolf, 1999). Previously, we showed that DRG neurons from animals that received a peripheral lesion $1 \mathrm{~d}$ before removal and culturing are no longer inhibited by MAG or myelin in general and that, 1 week after a peripheral lesion, not only is inhibition overcome but there is general improvement in growth (Qiu et al., 2002). We and others showed that the conditioning lesion effect is cAMP dependent and that injection of the cAMP analog db-cAMP directly into the DRG mimics the conditioning lesion effect in vivo (Neumann et al., 2002; Qiu et al., 2002). We also showed that, in overcoming inhibition by MAG, both a conditioning lesion and db-cAMP each trigger, first, a protein kinase A (PKA)-dependent and later a PKA-independent phase of growth, which then becomes transiently transcription dependent (Qiu et al., 2002; Gao et al., 2004). One of the genes that is upregulated in response to elevated cAMP is the enzyme arginase I (Arg I) (Cai et al., 2002). Arg I is key in the synthesis of the polyamine putrescine, which is 
readily converted to spermidine, which can then be converted, although not very readily, to spermine. In culture, either overexpression of Arg I in cerebellar neurons or addition of putrescine to the cultures is sufficient to overcome inhibition by MAG and by myelin in general (Cai et al., 2002). Now we report that upregulation of Arg I and increased synthesis of polyamines play an important role in the conditioning lesion effect, that putrescine must be converted to spermidine to overcome inhibitors of regeneration in myelin, and that spermidine is sufficient to promote optic nerve regeneration in vivo.

\section{Materials and Methods}

In vivo peripheral conditioning lesion. Postnatal day 18 (P18) to 21 rat pups were anesthetized by isofluorane, then a sciatic nerve was exposed at midthigh level, and a ligature was firmly tightened around the nerve distal to its emergence from the greater sciatic notch. The nerve was transected distal to the ligature, and the wound was closed. The animals were killed at the indicated times after the sciatic nerve transection.

Western blot analysis. L4 and L5 DRGs from the lesioned side and contralateral, unlesioned, control side were removed and lysed with radioimmunoprecipitation assay buffer $(150 \mathrm{~mm} \mathrm{NaCl}, 1.0 \%$ IGEPAL CA$630,0.5 \%$ sodium deoxycholate, $0.1 \%$ SDS, and $50 \mathrm{~mm}$ Tris, $\mathrm{pH} 8.0$ ) supplemented with phosphatase inhibitors $\left(1 \mathrm{~mm} \mathrm{Na} 3 \mathrm{VO}_{4}\right.$ and $1 \mathrm{~mm}$ $\mathrm{NaF}$ ) and proteinase inhibitors ( $1 \mathrm{~mm}$ EDTA, $1 \mathrm{~mm}$ PMSF, and aprotinin, leupeptin, and pepstatin at $1 \mu \mathrm{g} / \mathrm{ml}$ each). Protein concentration was measured with a Bio-Rad kit. Normalized lysates were boiled for $5 \mathrm{~min}$, after which they were subjected to SDS-PAGE in a $10 \%$ polyacrylamide gel, transferred to nitrocellulose membranes, and immunostained for Arg I (polyclonal) (Esch et al., 1998) at 1:5000 overnight at $4^{\circ} \mathrm{C}$. After two washes with PBS- $0.05 \%$ Tween 20 , the membranes were incubated with HRP-conjugated anti-rabbit IgG (1:5000) at room temperature for $1 \mathrm{~h}$. After an additional three washes with PBS-0.05\% Tween 20, HRP was visualized with ECL Western blotting reagent (GE Healthcare). The blot was stripped with stripping buffer ( $0.2 \mathrm{M}$ glycine, $\mathrm{pH} 2.2,1 \%$ Tween 20 , and $0.1 \%$ SDS) for $1 \mathrm{~h}$ at room temperature and reprobed with anti-actin (1:5000; Sigma).

Measurement of polyamines. Dissociated L4 and L5 DRG neurons at $1 \times 10^{6}$ or $5 \times 10^{6}$ cerebellar neurons from $\mathrm{P} 5$ rat pups were plated onto poly-L-lysine (PLL)-coated six-well plates. db-cAMP (1 mM) and/or biscyclohexylammonium sulfate (BCHS) $(200 \mu \mathrm{M})$ were added and incubated overnight. Cells were washed and lysed in $100 \mu \mathrm{l}$ of $1.5 \mathrm{M} \mathrm{HClO}_{4}$. Three lysates were pooled. Neutralizing buffer at $150 \mu \mathrm{l}$ ( $2 \mathrm{M}$ potassium bicarbonate) was added to stop the reaction. After centrifugation, polyamines in the supernatant were measured by HPLC performed by a service (Dr. G. Wu, Department of Animal Science, Texas A \& M University, College Station, TX). Glutamate concentration was measured in each sample as control. Data are presented as the concentration of polyamine per milliliter or the amount of polyamine per $1 \mu \mathrm{g}$ of glutamate.

Intrathecal administration. Osmotic minipumps (Alzet model 1007D, delivered at $0.5 \mu \mathrm{l} / \mathrm{h}$ ) were filled with putrescine $(12.5 \mathrm{nmol} / \mathrm{h})$, spermidine $(25 \mathrm{nmol} / \mathrm{h})$, difluoromethylornithine (DFMO) $(0.5 \mu \mathrm{mol} / \mathrm{h})$, BCHS (10 nmol/h) (all from Sigma), or vehicle as indicated, attached to a cannula, and then incubated at $37^{\circ} \mathrm{C}$ for at least $6 \mathrm{~h}$ to allow for release rate equilibration. P20-P23 rats were anesthetized with isofluorane, a laminectomy was performed to expose the spinal cord at the L 5 vertebral level, and an incision was made in the dura mater. The tip of the each cannula (attached to the minipump) was then guided into the subdural space, along the dorsal surface of the spinal cord, and secured in place by suture thread. Animals were killed between 1 and $7 \mathrm{~d}$ subsequently, and L4-L5 DRG neurons were isolated for the neurite outgrowth assay.

Neurite outgrowth assay on MAG or myelin. Monolayers of MAGexpressing or control $\mathrm{CHO}$ cells were grown to confluency in individual chambers of an eight-chamber tissue culture slide (Nalge Nunc International). Myelin was purified from rat CNS medulla (Norton and Poduslo, 1973), and $1 \mu \mathrm{g}$ of total protein per well was dried overnight onto the PLL-coated eight-chamber slides and used as a substrate (Shen et al., 1998). Dissociated DRG neurons or cerebellar neurons were resuspended in modified DMEM with Sato (200 nM progesterone, $224 \mathrm{nM}$ selenium, $4 \mu \mathrm{g} / \mathrm{ml}$ insulin, $0.35 \mathrm{mg} / \mathrm{ml} \mathrm{BSA}, 0.4 \mu \mathrm{g} / \mathrm{ml}$ L-thyroxine, and $0.34 \mu \mathrm{g} / \mathrm{ml}$ tri-iodo-thyronine). DRG neurons at $1.5 \times 10^{4}$ or $5 \times 10^{4}$ cerebellar neurons from either Long-Evans or Fischer rats were plated onto the $\mathrm{CHO}$ monolayers or myelin, and, after $18-20 \mathrm{~h}$, the neurons were fixed for $30 \mathrm{~min}$ with $4 \%$ paraformaldehyde (PFA) and permeabilized with ice-cold methanol for $2 \mathrm{~min}$. The cells were then blocked for 30 min with DMEM containing 10\% FCS and incubated overnight with a rabbit polyclonal antibody against either the growth-associated protein-43 (GAP-43) (1:4000; from R. Curtis and G. Wilkins, Imperial College, London, UK) or anti- $\beta$-III tubulin (1:1000, Tuj1; Covance Research Products) diluted in PBS-5\% BSA. Cells were washed three times with PBS and then incubated for $30 \mathrm{~min}$ at room temperature with a biotinylated donkey anti-rabbit or donkey anti-mouse IgG (1:500; GE Healthcare), respectively, washed three times, and then incubated with streptavidin-conjugated Texas Red (1:300; GE Healthcare) for $45 \mathrm{~min}$. After three more washes, the slides were mounted in Permafluor (Immunon) and viewed with a fluorescence microscope. The length of the longest neurite for each neuron for the first 200-600 neurons encountered when scanning the slide in a systematic manner was determined using an Oncor or MetaMorph image analysis program.

Optic nerve crush and intraocular injection. The optic nerve crush and the intraocular injection were performed as described previously (Leon et al., 2000) as follows. Male Fischer rats (250-300 g) were deeply anesthetized with isofluorane. The optic nerve was surgically exposed, the dural sheath surrounding the optic nerve was carefully incised, and the nerve was crushed with \#5 jewelers' forceps for $10 \mathrm{~s}$. The surgical site was sutured closed, and preservation of the central retinal artery was verified by direct ophthalmoscopy for signs of ischemic damage; animals showing signs of ischemic damage were excluded from the study. Immediately after the nerve crush, $10 \mu \mathrm{l}$ of saline, $20 \mu \mathrm{M}$ spermidine, or $40 \mu \mathrm{M}$ spermidine was injected intraocularly via a pulled-glass pipette using a nanoinjector (WPI). Recovery of postoperative animals was observed, and they were then individually housed with ad libitum access to food and water. Two weeks after surgery, animal were deeply anesthetized with ketamine/xylazine (100 and $10 \mathrm{mg} / \mathrm{kg}$, respectively) and transcardially perfused with $200 \mathrm{ml}$ of heparinized saline (1000 U/l) and $300 \mathrm{ml}$ of $4 \%$ PFA. The optic nerve was dissected out, postfixed in $4 \%$ PFA overnight, and cryoprotected in $30 \%$ sucrose in Tris-buffered saline (TBS). Additionally the lens of each eye was examined for injury (opaque eyes) at the time of removal - nerves from eyes exhibiting such injury were excluded from the study.

Immunohistochemistry and image analysis. Frozen serial sections (20 $\mu \mathrm{m})$ were cut from the optic nerves described above and immunofluorescent labeling was performed as follows: sections were washed $4 \mathrm{x}$ with TBS, blocked for $1 \mathrm{~h}$ with TBS plus $0.2 \%$ Triton X-100 plus $5 \%$ normal goat serum and then incubated with sheep anti-GAP-43 primary antibody (gift from L. Benowitz, Children's Hospital, Boston, MA), diluted 1:100 in blocking buffer, overnight at $4^{\circ} \mathrm{C}$. Sections were then washed four times with TBS and incubated with goat anti-sheep conjugated to FITC, diluted 1:500 in blocking buffer, for $1 \mathrm{~h}$ at room temperature. Finally, sections were washed four times with TBS and coverslipped with aqueous mounting medium before imaging. Sections were scanned using a Nikon Eclipse 90i microscope at $20 \times$ magnification, using MetaMorph acquisition software to stitch together a single high-resolution image. The distance traversed by the three longest GAP-43-positive axons, relative to the distal edge of the lesion, was measured for each animal. Four animals were included in each group.

Data analysis. Prism GraphPad software was used to perform one-way ANOVA, followed by multiple comparisons using the Tukey's procedure.

\section{Results}

Arg I and synthesis of polyamines are upregulated in DRG neurons in response to a peripheral lesion

Previously, we showed that cAMP upregulates expression of Arg I and that overexpression of Arg I, in either cerebellar or DRG neurons, or addition of putrescine were each sufficient to overcome inhibition by MAG and myelin (Cai et al., 2002). Now we wanted to determine whether upregulation of Arg I and synthesis 


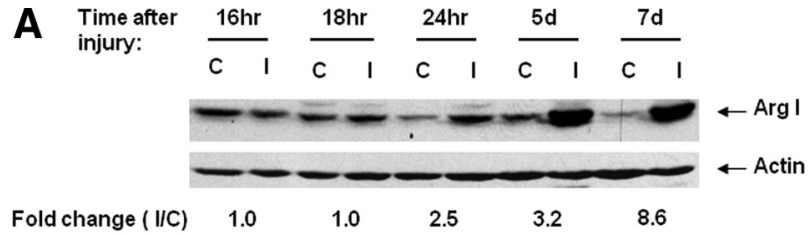

B

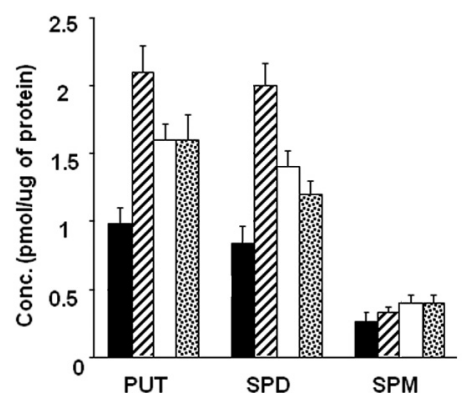

Figure 1. Arginase l and polyamines are upregulated by a peripheral, conditioning lesion. $\boldsymbol{A}$, At various times, as indicated, after a unilateral, peripheral lesion to the sciatic nerve the DRGs were removed and subjected to Western blotting and stained for Arg I (I). The contralateral, uninjured DRGs were used as a control (C). Total protein at $50 \mu \mathrm{g}$ were loaded per well. Blots were stripped and reprobed for actin as a loading control. $\boldsymbol{B}$, DRGs were removed at $2 \mathrm{~d}$ (striped bars), $4 \mathrm{~d}$ (white bars), or $7 \mathrm{~d}$ (confetti bars) after a peripheral lesion or unlesioned (black bars) and cultured overnight before the cells were lysed and subjected to HPLC analysis for the polyamines putrescine (PUT), spermidine (SPD), and spermine (SPM).

of polyamines contributed to the peripheral, conditioning lesion effect. We first asked whether expression of Arg I protein increased after a conditioning lesion. Sciatic nerves were lesioned and the animals were killed after different times, the DRGs were removed, and Western immunoblotting was performed for Arg I. The contralateral, uninjured DRG neurons from the animals were used as a control. Figure $1 A$ shows that an increase in Arg I protein is first observed at $24 \mathrm{~h}$ after lesion and increases even further by 5 and $7 \mathrm{~d}$ after lesion. At all of the times after lesion, under these conditions, Arg I levels are low in the DRG neurons from the unlesioned nerves.

To assess whether the upregulation of Arg I subsequent to a peripheral lesion results in an increase in polyamine synthesis, sciatic nerves were lesioned unilaterally and DRGs were removed from the animals 2, 4, and $8 \mathrm{~d}$ later. The DRG neurons were cultured overnight, then the cells were washed and lysed, and the three polyamines putrescine, spermidine, and spermine were measured by HPLC. At $2 \mathrm{~d}$ after lesion, both putrescine and spermidine had more than doubled, whereas spermine was unchanged. By 4 and $8 \mathrm{~d}$, putrescine and spermidine had decreased slightly, but levels were still significantly higher than in DRGs from unlesioned nerves. Spermine levels did not change even at 4 or $8 \mathrm{~d}$ after lesion (Fig. $1 \mathrm{~B}$ ). The increased synthesis of putrescine and spermidine is consistent with an increase in expression of Arg I after a conditioning lesion and could therefore play a role in the conditioning lesion effect on neurite outgrowth on MAG.

\section{The conditioning lesion effect is initially dependent on ongoing polyamine synthesis but with time becomes independent}

An inhibitor of the polyamine synthesis pathway is DFMO, which blocks ornithine decarboxylase, the enzyme following Arg I in the polyamine synthesis pathway. To determine whether the improved growth on MAG and myelin after a conditioning lesion is dependent on this pathway, DFMO was included in the cultures when the DRG neurons were removed from the animals. At $18 \mathrm{~h}$ after lesion, the ability of DRG neurons from lesioned nerves to overcome inhibition by MAG was completely blocked by DFMO (Fig. 2). DFMO had no effect on neurons grown on control CHO cells, not expressing MAG. In sharp contrast, DFMO had no effect when added to the cultures of DRG neurons that were removed from the animal $24 \mathrm{~h}$ (Fig. 2) or 1 week (data not shown) after a conditioning lesion; there was no inhibition by MAG, and there was also a general improvement in growth on control cells (Fig. 2). These results imply that, at $24 \mathrm{~h}$ after lesion, ongoing synthesis of polyamines is not required for the neurons to extend neurites in the presence of MAG. It is of note that ongoing polyamine synthesis is required at $18 \mathrm{~h}$ after lesion, but there is no detectable increase in Arg I at this time, and the neurite outgrowth assay takes an additional $18 \mathrm{~h}$, which is equivalent to $36 \mathrm{~h}$ after lesion. Therefore, during the culture period, at the equivalent of $24 \mathrm{~h}$ after lesion, Arg I expression increases and the ability to grow in an inhibitory environment becomes dependent on ongoing polyamine synthesis. Hence, the time when the increase in Arg I expression first occurs appears to be the crucial time when ongoing polyamine synthesis is required to overcome inhibition when the neurons are cultured. Neurons removed at $24 \mathrm{~h}$ after lesion are then cultured for the next $18 \mathrm{~h}$, equivalent to $42 \mathrm{~h}$ after lesion, a time when Arg I expression is already increased and sufficient polyamine has been synthesized and/or a signaling pathway initiated to overcome inhibition such that ongoing synthesis is no longer necessary.

\section{Intrathecal delivery of an inhibitor of the polyamine pathway} attenuates both the early and the late phases of growth triggered by a peripheral lesion

To ask whether the later phase of growth that is independent of ongoing polyamine synthesis requires a previous increase in polyamine synthesis, DFMO was delivered, continuously, intrathecally for the entire time between when the lesion was performed and when the DRG neurons were removed from the animals. When DRG neurons from animals that received DFMO continuously for the $24 \mathrm{~h}$ after a conditioning lesion are removed, they are still inhibited by MAG and the DFMO has no effect on the ability of neurons to extend neurites on control CHO cells not expressing MAG (Fig. 3); the ability to overcome inhibition is blocked but not general growth. By 1 week after lesion with DFMO delivered continuously, the ability of DRG neurons to extend neurites on MAG is significantly reduced. However, the general improvement in growth on control CHO cells is unaffected by the presence of DFMO (Fig. 3). This suggests that, although the ability of a conditioning lesion to overcome inhibition by MAG 1 week subsequent to a conditioning lesion does not require ongoing polyamine synthesis, it does require a previous increase in synthesis in vivo.

\section{The ability of db-cAMP to overcome inhibition by MAG is} dependent on the conversion of putrescine to spermidine Previously, we reported that, like a conditioning lesion, db-cAMP upregulates expression of Arg I. Consistent with this, we now show that treatment of cerebellar neurons with db-cAMP results in an increase in synthesis, by approximately twofold, of the polyamines putrescine and spermidine but not of spermine (Fig. 4A). In cells, putrescine is readily converted to spermidine, which then can be converted, but not very readily, to spermine. To determine whether the increased synthesis of putrescine itself is sufficient for $\mathrm{db}$-cAMP to overcome inhibition by MAG or whether it must be converted to one of the two other polyamines, the enzyme that converts putrescine to spermidine, spermidine synthase, was 
blocked with the specific inhibitor BCHS. Figure $4 A$ shows that the inhibitor is working and does indeed block the conversion of putrescine to spermidine because, when added to cerebellar neurons even without treatment with db-cAMP, putrescine accumulates and the basal levels of spermidine and spermine remain unchanged. When BCHS is added along with db-cAMP again, putrescine accumulates but now the increase in spermidine is blocked (Fig. 4A). To determine whether putrescine must be converted to spermidine for db-cAMP to overcome inhibition by MAG, BCHS was added along with $\mathrm{db}$-cAMP in the neurite outgrowth assay. Figure $4 B$ (and supplemental Table 1 , available at www.jneurosci.org as supplemental material) shows that, as we reported previously, db-cAMP overcomes inhibition by MAG. Now we show that BCHS blocks this effect; BCHS alone has no effect on inhibition by MAG. These results indicate that putrescine must be converted to spermidine to overcome inhibition by MAG. In addition, with either condition, db-cAMP alone or when combined with BCHS, there is no change in the levels of spermine, strongly suggesting that spermine plays no role in this effect and that it is spermidine rather than putrescine that is responsible for overcoming the inhibition of MAG.

\section{In vivo putrescine must be converted to spermidine to overcome inhibition by MAG}

To first determine whether putrescine delivered intrathecally could overcome inhibition by MAG, the polyamine was delivered continuously for $4 \mathrm{~d}$ before the DRG neurons were removed and assessed for their ability to grow in an inhibitory environment. As can be seen in Figure 5, after $4 \mathrm{~d}$ of putrescine delivery, not only are DRG neurons no longer inhibited by MAG but there is also general improvement in growth on the control $\mathrm{CHO}$ cells not expressing MAG, a result similar to that observed after the conditioning lesion (Fig. 5). However, when BCHS is delivered along with putrescine for $4 \mathrm{~d}$, putrescine has no effect; neurite outgrowth from DRG neurons is still inhibited by MAG, and there is no general improvement in growth on the control cells (Fig. 5). These results demonstrate that in vivo putrescine must be converted to spermidine to block inhibition by MAG when the neurons are subsequently cultured.

\section{Spermidine overcomes inhibition by MAG and myelin in a} dose-dependent manner

Above we have shown that putrescine must be converted to spermidine, both in vivo and in culture, to overcome inhibition by MAG. Now we show that spermidine can overcome inhibition by MAG and myelin in a dose-dependent manner. Figure 6 shows that, when DRG neurons are incubated with various concentrations of spermidine overnight before they are cultured on MAGexpressing cells, control cells, or myelin, a treatment we term "priming," inhibition is overcome in a dose-dependent manner. At a concentration of $20 \mu \mathrm{M}$, spermidine is as effective as dbcAMP in overcoming inhibition by both MAG and myelin, at 100 $\mu \mathrm{M}$ this effect is diminished, and at $500 \mu \mathrm{M}$ it is completely lost. We found that priming with spermidine is more effective than if

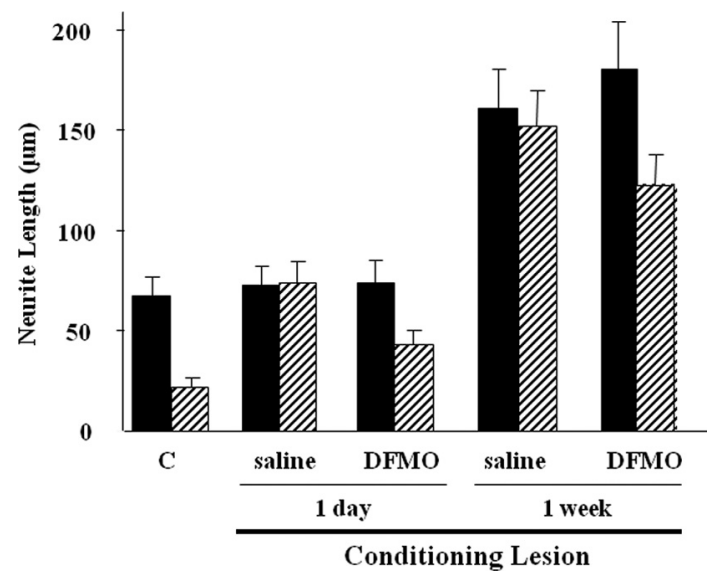

Figure 3. Inhibition of polyamine synthesis in vivo attenuates both the ongoing polyamine synthesis-dependent and -independent growth triggered by a peripheral lesion. At the same time as the sciatic nerve was lesioned, intrathecal minipumps were inserted that delivered 0.5 $\mu \mathrm{mol} / \mathrm{h} \mathrm{DFMO}$ for the entire time until the animals were killed, and the DRGs were removed and cultured on either MAG-expressing (striped bars) or control CHO (black bars) cells. Saline was delivered intrathecally as a control, and results were compared with neurite outgrowth of DRG neurons from quantitation of neurite outgrowth. Results represent the length of the longest neurite from $\sim 200$ neurons \pm SEM, as percentage of neurite length of neurons from unlesioned, contralateral controls grown on control $\mathrm{CHO}$ cells. C, Control.

spermidine is added directly to the cultures (results not shown). These results show not only that spermidine is sufficient and as effective as db-cAMP in overcoming inhibition by MAG and myelin but also, because it is effective with priming, it does not have to be present during the neurite outgrowth assay; the "pathway" triggered in neurons by exposure to spermidine is sufficient to subsequently overcome inhibition.

\section{Intrathecal delivery of spermidine overcomes inhibition by MAG}

To determine whether spermidine delivered in vivo is sufficient to overcome inhibition by MAG and myelin when DRG neurons are subsequently cultured, spermidine was delivered continuously, 
A

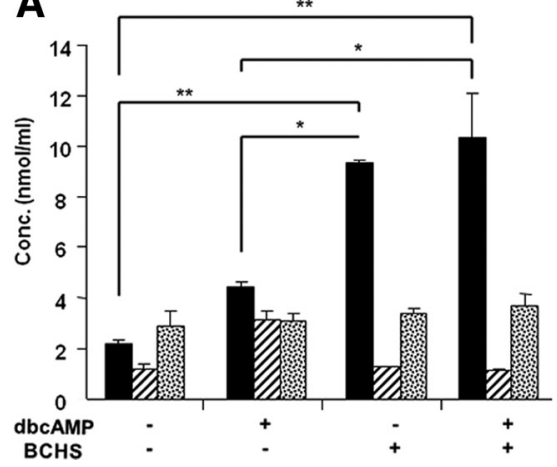

B

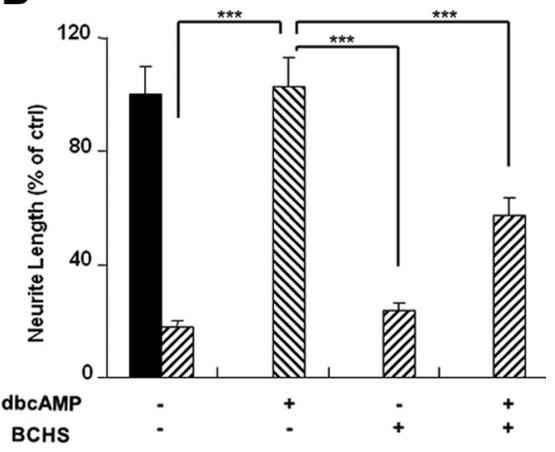

Figure 4. To overcome inhibition by MAG, putrescine must be converted to spermidine. $A$, Dissociated cerebellar neurons from postnatal day 5 rats were removed and cultured overnight with and without $\mathrm{db}$-CAMP $(1 \mathrm{mM})$, in the presence or absence of BCHS (200 $\mu \mathrm{M}$ ) before being lysed, and the lysates were analyzed by HPLC for the polyamines putrescine (black bars), spermidine (striped bars), and spermine (confetti bars). $\boldsymbol{B}$, Cerebellar neurons were removed and cultured overnight on either MAG-expressing CHO cells (striped bars) or control CHO cells (black bars), with and without db-CAMP (1 mM), in the presence or absence of BCHS (200 $\mu \mathrm{m}$ ) before being fixed and stained for $\beta$-III tubulin. Results represent the length of the longest neurite from $\sim 400$ neurons \pm SEM. ${ }^{*} p \geq 0.05 ;{ }^{* *} p \geq 0.01 ;{ }^{* * *} p \geq 0.001$.

A

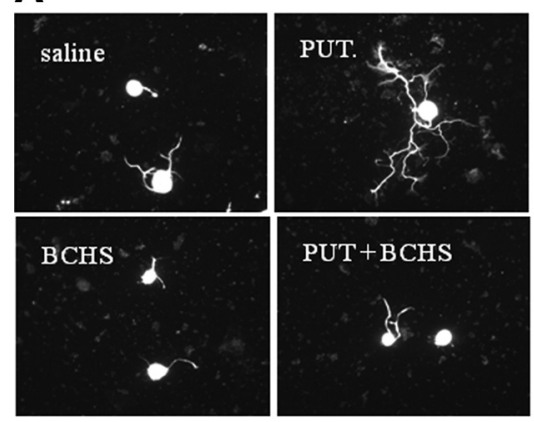

B

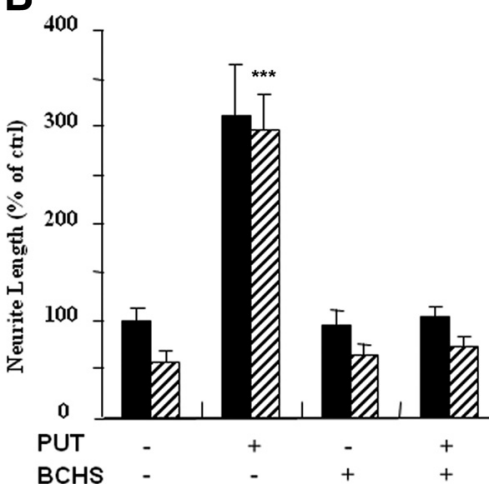

Figure 5. In vivo putrescine must be converted to spermidine to overcome inhibition by MAG. C, Putrescine (PUT) $(12.5 \mathrm{nmol} / \mathrm{h})$ with and without BCHS $(10 \mathrm{nmol} / \mathrm{h})$ were delivered intrathecally for $4 \mathrm{~d}$ before the DRG neurons were removed and cultured overnight on either MAG-expressing CHO cells (striped bars) or control CHO cells (black bars), after which they were fixed and stained for $\beta$-III tubulin. $\boldsymbol{A}$, Representative images of neurons growing on MAG cells under various conditions as indicated. $\boldsymbol{B}$, Quantitation of neurite length for the longest neurite from $\sim 200$ neurons \pm SEM. ${ }^{* * *} p \geq 0.001$ compared with neurite length on MAG cells.

A

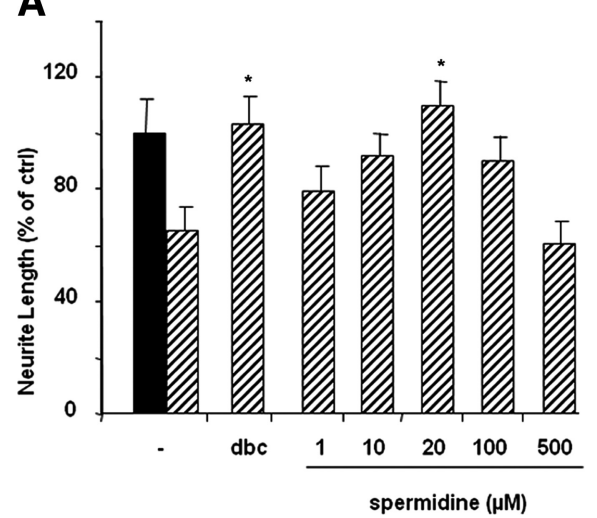

B

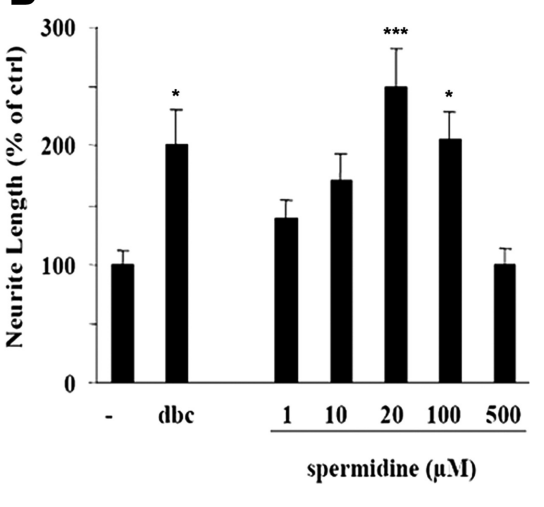

Figure 6. Priming neurons with spermidine overcomes inhibition by MAG and myelin in a dose-dependent manner. $\boldsymbol{A}, \boldsymbol{B}, \mathrm{DRG}$ neurons were incubated overnight with various concentrations of spermidine as indicated or $1 \mathrm{~mm} d \mathrm{~b}$-cAMP (dbc) before being cultured overnight on either MAG-expressing $\mathrm{CHO}$ cells (striped bars) or control CHO cells (black bars) $(\boldsymbol{A})$ or myelin ( $\boldsymbol{B})$, after which they were fixed and stained for $\beta$-III tubulin. Quantitation of neurite length for the longest neurite from $\sim 200$ neurons \pm SEM. ${ }^{*} p \geq 0.05,{ }^{* * *} p \geq 0.001$ compared with neurite length on MAG cells $(\boldsymbol{A})$ or myelin $(\boldsymbol{B})$.

intrathecally for $4 \mathrm{~d}$ at a dose of 25 nmol/h. Figure 7 shows that DRG neurons from animals that received spermidine intrathecally are no longer inhibited by MAG. Furthermore, DRG neurons from these spermidine-treated animals also showed a general improvement in growth on the control $\mathrm{CHO}$ cells. Likewise, when DRG neurons from the spermidine-treated animals are grown on myelin, they grow three times longer. These results are consistent with the ability of spermidine to overcome inhibition when neurons are primed with the polyamine. They also show that intrathecal delivery of spermidine is sufficient to mimic the conditioning lesion effect on the ability of neurons to grow in an inhibitory environment.

\section{Spermidine promotes optic nerve regeneration in vivo}

To assess whether spermidine can promote CNS axon regeneration in vivo, a simple model of axon regeneration, the optic nerve model, was used as a proof-ofprinciple. At the same time as the optic nerve was crushed at $2 \mathrm{~mm}$ behind the eye, a single injection of spermidine at 20 or 40 $\mu \mathrm{M}(10 \mu \mathrm{l})$ was administered intraocularly. Great care was taken not to damage the lens because the resulting inflammation in itself can promote optic nerve regeneration (Leon et al., 2000). Those animals in which there was lens injury were not included in the analysis. Two weeks after lesion, the animals were killed and the optic nerves were stained for GAP-43, which is only upregulated in severed axons. We found that only at $20 \mu \mathrm{M}$ did spermidine have a significant effect on regeneration. Figure $8 \mathrm{~A}$ shows the results from two typical animals, one that received $20 \mu \mathrm{M}$ spermidine (top) and one that received saline (bottom). Regenerated nerves up to $4 \mathrm{~mm}$ beyond the lesion site are clearly apparent in the spermidine-treated but not the vehicletreated control animal. Figure $8 B$ shows that, for $20 \mu \mathrm{M}$-treated but not $40 \mu \mathrm{M}$ treated animals, there is a statistically significant increase in optic nerve axon regeneration in the spermidine-treated animals compared with vehicle-treated animals. Furthermore, the number of axons in the spermidine-treated animals that reached between 500 and $2500 \mu \mathrm{m}$ past the lesion site was on average 122 per animal, whereas for vehicle-treated animal, the average per animal was 42 and no axons in the control animals ever reached $2500 \mu \mathrm{m}$. Although the number of regenerating axons is relatively low, most likely 


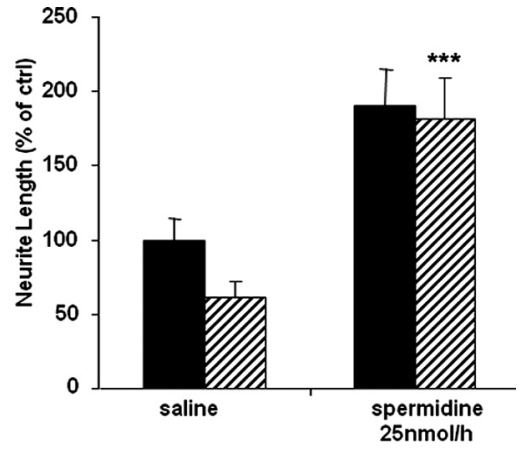

Figure 7. Intrathecal delivery of spermidine overcomes inhibition by MAG and myelin when neurons are cultured. Spermidine was delivered via intrathecal minipumps at a rate of $25 \mathrm{nmol} / \mathrm{h}$ for $4 \mathrm{~d}$ before the DRG neurons were removed and cultured overnight on either MAG-expressing $\mathrm{CHO}$ cells (striped bars) or control $\mathrm{CHO}$ cells (black bars), after which they were fixed and stained for $\beta$-III tubulin. Quantitation of neurite length for the longest neurite from $\sim 200$ neurons \pm SEM. ${ }^{* * *} p \geq 0.001$ compared with neurite length on MAG cells.

\section{A}
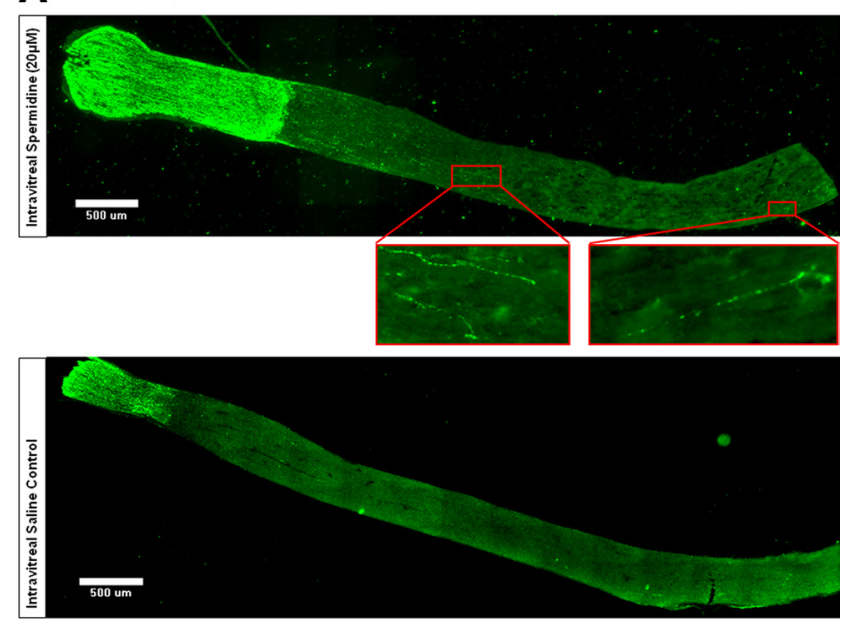

B

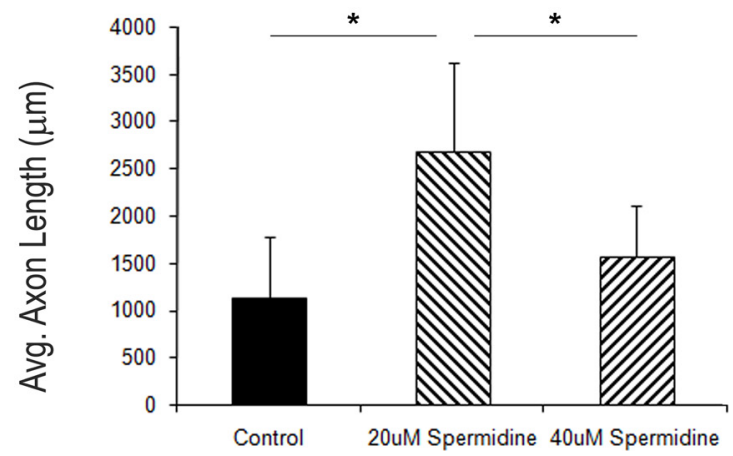

Figure 8. Spermidine promotes optic nerve regeneration in vivo. At the same time as the optic nerve was crushed at $2 \mathrm{~mm}$ behind the eye, a single injection of $10 \mu \mathrm{l}$ of spermidine at either 20 or $40 \mu \mathrm{m}$ or saline was delivered intraocularly. Two weeks later, the animals were killed, and the optic nerves removed, fixed, sectioned, and stained for GAP-43. A, Representative images from two typical animals: top, $20 \mu$ m spermidine; bottom, saline. Boxed areas show higher magnification of axons that had regenerated $\sim 2$ and $4 \mathrm{~mm}$ beyond the lesion site. $\boldsymbol{B}$, Quantitation of the distance regenerated beyond the lesion site for the three longest axons for each animal. Four animals per group. ${ }^{*} p \geq 0.05$.

because we have not maximized the effect, there are still more axons regenerating in the spermidine-treated animals. These results show that spermidine is sufficient to promote axon regeneration in the adult CNS.

\section{Discussion}

Our ultimate goal is to identify the molecules that change after a peripheral, conditioning lesion that allow subsequently lesioned spinal axons to grow in an inhibitory environment and hence regenerate in vivo. By doing this, we hope to be able to mimic with molecular tools the effects of a conditioning lesion on regeneration in vivo without performing a peripheral lesion, which of course cannot be used as a treatment to encourage spinal axon regeneration in humans. Now we propose that the upregulation of Arg I and the consequential increased synthesis of spermidine are likely to be major contributors to this effect. We show the following: that increases in both Arg I expression and synthesis of spermidine are sustained up to $7 \mathrm{~d}$ after peripheral lesion; that if the synthesis of polyamines is blocked in vivo, the effects of a conditioning lesion on the ability of neurons to grow in an inhibitory environment when subsequently cultured is blocked at $1 \mathrm{~d}$ after lesion and attenuated at 1 week; that putrescine, either delivered directly in culture or delivered in vivo, must be converted to spermidine to allow neurons to grow in an inhibitory environment; that intrathecal delivery of spermidine is sufficient to mimic the conditioning lesion effect when neurons are cultured; and that spermidine is sufficient to promote optic nerve regeneration when it is delivered intraocularly at the same time as the lesion.

These results are important for a number of reasons. First, previously, we showed that the conditioning lesion effect both in culture and in vivo could be mimicked by elevating cAMP using db-cAMP (Qiu et al., 2002). We also showed that the db-cAMP effect in overcoming inhibition by MAG/myelin in culture was dependent on upregulation of Arg I and synthesis of polyamines (Cai et al., 2002). Now we show that this holds true for the conditioning lesion effect as well. More precisely, we have identified spermidine, not putrescine, as the polyamine responsible for these effects. Spermidine, then, represents a more precise and specific target than cAMP for intervention and drug development to promote axonal regeneration in humans. Because it is highly unlikely that spermidine activates as many pathways as elevation of cAMP, the activation of pathways not required for regeneration, some of which may be detrimental, would be reduced. Second, spermidine had an effect when it was delivered at the same time as the lesion was performed. It was not delivered before the lesion as we had shown was the case for db-cAMP. This too makes spermidine a more attractive possible treatment because it is impossible to treat prophalactically for spinal cord or brain injury. Third, spermidine was effective when delivered to the cell bodies of the retinal ganglion cells, which means it does not have to be delivered directly to the lesion site per se to encourage regeneration. This could make a significant difference in effective delivery because neuronal cell bodies, for example of cortico-spinal tract axons, are more readily accessible than delivery to the injured spinal cord. Finally, spermidine did not have to be present constantly to promote regeneration. Only a single dose to the retinal ganglion cell bodies resulted in regeneration of optic nerve, and, after intrathecal delivery, the effect of spermidine is sustained when the neurons are removed and subsequently cultured in the absence of the polyamine. This strongly suggests that spermidine is triggering the activation of a pathway that overcomes inhibition. It is of note, however, that, because we have only delivered a single dose of spermidine, the effect on optic nerve regeneration is unlikely to be optimal and/or maximized. Here, we have only shown a proof-of-principle that spermidine can promote CNS axonal regeneration in vivo. 
Previously, we reported that a conditioning lesion triggered two phases of growth an initial phase that was PKA dependent and a later phase of growth that was PKA independent and that a transient increase in CAMP was sufficient to elicit both phases (Qiu et al., 2002). Now we extend this model to suggest that increased expression of Arg I after a conditioning lesion also triggers two phases of growth: an early phase that is dependent on ongoing polyamine synthesis and a later phase that is independent of ongoing polyamine synthesis. We reach this conclusion because blocking ongoing polyamine synthesis in culture of neurons from animals that received a peripheral lesion $18 \mathrm{~h}$ before blocks completely their ability to overcome inhibition by MAG, but, for animals that received the peripheral lesion $24 \mathrm{~h}$ or 1 week before the DRG neurons were removed, blocking polyamine synthesis in culture has no effect on either the ability to overcome inhibition by MAG and also the general improvement in growth even on the control, permissive CHO cells. However, we also propose that an increase in Arg I expression and an increase in polyamine synthesis is necessary for the later phase of growth triggered by a conditioning lesion, which is independent of ongoing polyamine synthesis for a number of reasons. First, if the polyamine pathway is blocked by intrathecal delivery of DFMO for the entire time between when the peripheral nerve is lesioned and the DRG neurons are removed and cultured, at $1 \mathrm{~d}$ after lesion, the ability to overcome inhibition by MAG is completely blocked and, at 1 week, it is attenuated. Second, both putrescine and spermidine delivered intrathecally can each not only allow the DRG neurons to grow in the presence of MAG when cultured but also induce an improvement in general growth. The reason that intrathecal delivery of DFMO does not completely block the conditioning lesion effect at $7 \mathrm{~d}$ after lesion could be attributable to incomplete block of the pathway for the entire $7 \mathrm{~d}$, perhaps because of delivery problems, whereas more effective delivery and blockade may have been achieved when delivered for only $1 \mathrm{~d}$. Alternatively, events other than ongoing or previous polyamine synthesis may contribute to the good growth at $7 \mathrm{~d}$ but not at $1 \mathrm{~d}$ after lesion. This is likely to be the case because we have already reported that upregulation of other proteins, such as IL-6, in parallel to Arg I occur after a conditioning lesion and that, like polyamines, IL-6 is sufficient to mimic the conditioning lesion effect and promote regeneration in vivo (Cao et al., 2006). However, unlike polyamines, we believe that IL- 6 is sufficient but not necessary for the conditioning lesion effect because the response of neurons from IL-6 knock-out animals, both in culture and in vivo, is indistinguishable from neurons from wild-type mice (Cao et al., 2006). Conversely, we also identified other proteins that are upregulated after a conditioning lesion, also in parallel to Arg I, which appear to be both necessary and sufficient for the conditioning lesion effect (our unpublished observation). We propose that a number of parallel pathways are triggered by a conditioning lesion (and by cAMP) but that each is activated below the threshold necessary to alone elicit a conditioning lesion effect; however, when activated together, they reach this threshold. This in turn could explain why blocking any single pathway blocks the conditioning lesion effect (threshold is not reached) (e.g., Arg I), whereas activating any one pathway maximally is sufficient to alone mimic the conditioning lesion effect (threshold is reached) (e.g., IL-6 or Arg I). Other genes that have been reported to increase with a conditioning lesion, such as the transcription factor ATF3, can promote general growth but do not overcome inhibitors of regeneration in myelin and are neither necessary nor sufficient for the conditioning lesion effect (Seijffers et al., 2006, 2007).

Almost two decades ago, polyamines were reported to have an effect on brain development (Slotkin and Bartolome, 1986), on the rate of peripheral nerve regeneration (Gilad et al., 1996), on neuritogenesis (Dornay et al., 1986), and on neuronal survival (Gilad and Gilad, 1988). We now add to the list of attributes of these small molecules: they promote axonal regeneration in the inhibitory environment of the adult CNS. Polyamines have been reported to have a plethora of effects on many aspects of neuronal behavior from binding ion channels (Williams, 1997), to affecting the dynamics of the cytoskeleton (Kamińska et al., 1992; Banan et al., 1998), to posttranslationally modifying proteins (Chakraborty et al., 1987; Huang et al., 2007). The challenge now is to identify which of these mechanisms spermidine is triggering to bring about this regeneration.

\section{References}

Atwal JK, Pinkston-Gosse J, Syken J, Stawicki S, Wu Y, Shatz C, TessierLavigne M (2008) PirB is a functional receptor for myelin inhibitors of axonal regeneration. Science 322:967-970.

Banan A, McCormack SA, Johnson LR (1998) Polyamines are required for microtubule formation during gastric mucosal healing. Am J Physiol 274:G879-G885.

Cai D, Deng K, Mellado W, Lee J, Ratan RR, Filbin MT (2002) Arginase I and polyamines act downstream from cyclic AMP in overcoming inhibition of axonal growth MAG and myelin in vitro. Neuron 35:711-719.

Cao Z, Gao Y, Bryson JB, Hou J, Chaudhry N, Siddiq M, Martinez J, Spencer T, Carmel J, Hart R, Mellado W, Filbin MT (2006) The cytokine, IL-6, is sufficient but not necessary to mimic the peripheral conditioning lesion effect on axonal growth. J Neurosci 26:5565-5573.

Chakraborty G, Leach T, Zanakis MF, Sturman JA, Ingoglia NA (1987) Posttranslational protein modification by polyamines in intact and regenerating nerves. J Neurochem 48:669-675.

Dornay M, Gilad VH, Shiler I, Gilad GM (1986) Early polyamine treatment accelerates regeneration of rat sympathetic neurons. Exp Neurol 92:665-674.

Esch F, Lin KI, Hills A, Zaman K, Baraban JM, Chatterjee S, Rubin L, Ash DE, Ratan RR (1998) Purification of a multipotent antideath activity from bovine liver and its identification as arginase: nitric oxideindependent inhibition of neuronal apoptosis. J Neurosci 18:4083-4095.

Filbin MT (2003) Myelin-associated inhibitors of axonal regeneration in the adult mammalian CNS. Nat Rev Neurosci 4:703-713.

Gao Y, Deng K, Hou J, Bryson JB, Barco A, Nikulina E, Spencer T, Mellado W, Kandel ER, Filbin MT (2004) Activation of CREB is necessary and sufficient to overcome myelin inhibitors and encourage spinal axon regeneration in vivo. Neuron 44:609-621.

Gilad GM, Gilad VH (1988) Early polyamine treatment enhances survival of sympathetic neurons after postnatal axonal injury or immunosympathectomy. Brain Res 466:175-181.

Gilad VH, Tetzlaff WG, Rabey JM, Gilad GM (1996) Accelerated recovery following polyamines and aminoguanidine treatment after facial nerve injury in rats. Brain Res 724:141-144.

Huang Y, Higginson DS, Hester L, Park MH, Snyder SH (2007) Neuronal growth and survival mediated by eIF5A, a polyamine-modified translation initiation factor. Proc Natl Acad Sci U S A 104:4194-4199.

Kamińska B, Kaczmarek L, Grzelakowska-Sztabert B (1992) Inhibitors of polyamine biosynthesis affect the expression of genes encoding cytoskeletal proteins. FEBS Lett 304:198-200.

Leon S, Yin Y, Nguyen J, Irwin N, Benowitz LI (2000) Lens injury stimulates axon regeneration in the mature rat optic nerve. J Neurosci 20:4615-4626.

Neumann S, Woolf CJ (1999) Regeneration of dorsal column fibers into and beyond the lesion site following adult spinal cord injury. Neuron 23:83-91. 
Neumann S, Bradke F, Tessier-Lavigne M, Basbaum AI (2002) Regeneration of sensory axons within the injured spinal cord induced by intraganglionic cAMP elevation. Neuron 34:885-893.

Norton WT, Poduslo SE (1973) Myelination in rat brain: method of myelin isolation. J Neurochem 21:749-757.

Qiu J, Cai D, Dai H, McAtee M, Hoffman PN, Bregman BS, Filbin MT (2002) Spinal axon regeneration induced by elevation of cyclic AMP. Neuron 34:895-903.

Seijffers R, Allchorne AJ, Woolf CJ (2006) The transcription factor ATF-3 promotes neurite outgrowth. Mol Cell Neurosci 32:143-154.

Seijffers R, Mills CD, Woolf CJ (2007) ATF3 increases the intrinsic growth state of DRG neurons to enhance peripheral nerve regeneration. J Neurosci 27:7911-7920.
Shen YJ, DeBellard ME, Salzer JL, Roder J, Filbin MT (1998) Myelinassociated glycoprotein in myelin and expressed by Schwann cells inhibits axonal regeneration and branching. Mol Cell Neurosci 12:79-91.

Silver J, Miller JH (2004) Regeneration beyond the glial scar. Nat Rev Neurosci 5:146-156.

Slotkin TA, Bartolome J (1986) Role of ornithine decarboxylase and the polyamines in nervous system development: a review. Brain Res Bull 17:307-320.

Williams K (1997) Interactions of polyamines with ion channels. Biochem J 325:289-297.

Yiu G, He Z (2006) Glial inhibition of CNS axon regeneration. Nat Rev Neurosci 7:617-627. 